PROCEEDINGS OF THE

AMERICAN MATHEMATICAL SOCIETY

Volume 97, Number 3, July 1986

\title{
PICARD AND BRAUER GROUPS OF ZARISKI SCHEMES
}

\author{
PIOTR BLASS ${ }^{1}$ AND RAYMOND HOOBLER
}

\begin{abstract}
The Cartier-Yuan exact sequence is used to calculate Picard groups and Brauer groups of Zariski surfaces and their generalizations. A result of Blass-Deligne on the factoriality of general affine Zariski surfaces is extended to all higher dimensional Zariski schemes.
\end{abstract}

In this note we use the Cartier-Yuan exact sequence to calculate Picard groups and Brauer groups of Zariski surfaces and their generalizations. We also extend a result of Blass-Deligne on the factoriality of general affine Zariski surfaces to all higher dimensional Zariski schemes. All calculations are carried out using étale cohomology but at several points we use the identification of étale and Zariski cohomology if the coefficient sheaf is a coherent $O_{S}$-module.

Since we are interested in both the affine and projective cases we begin by establishing some notation. Let $S$ be a regular, quasi-projective scheme in characteristic $p>0$ with very ample bundle $O(1)$. If $e$ is a positive integer, $(p, e)=1$, and $G \in \Gamma\left(S, O_{S}(p e)\right)$, we let $\underline{A}$ be the graded, quasi-coherent sheaf of $O_{S}$-algebras defined by $\underline{A}=\bigoplus_{l=0}^{\infty} O(l)[Z] /\left(Z^{p}-G\right)$ where $Z$ is an indeterminate of degree $e$. The Zariski scheme associated to $(S, G)$ is $X=\operatorname{Proj}(\underline{A})$. It comes with a structure map $\pi: X \rightarrow S$ which makes $\pi_{*} O_{X}$ into a locally free $O_{S}$-module of rank $p$. Note that if $S$ is affine, then $X=\operatorname{Spec}\left(O_{S}[Z] /\left(Z^{p}-G\right)\right)$.

For the remainder of this note we assume that $X$ is a normal scheme. This will be the case, for instance, if $S$ is a smooth, quasi-projective variety and the locus of zeros of the Jacobian matrix of $G / x_{j}^{p e}$ has codimension $>1$ in $D_{+}\left(x_{j}\right) \subset S$ and all $x_{j} \in \Gamma(S, O(1))$. In this case,

$$
\pi_{*} O_{X}=\bigoplus_{l=0}^{p-1} O_{S}(-l e)
$$

since the section $Z$ restricts to $Z_{j}=Z / x_{j}^{e} \in \Gamma\left(D_{+}\left(x_{j}\right), \pi_{*} O_{X}\right)$ which transforms according to the rule

$$
\left(Z_{j} / Z_{k}\right)^{p}=\left(G / x_{j}^{p e}\right) /\left(G / x_{k}^{p e}\right)=\left(x_{k}^{e} / x_{j}^{e}\right)^{p}
$$

or, more simply, $x_{j}^{e} Z_{j}=x_{k}^{e} Z_{k}$. Moreover, the Frobenius on $S, F_{S}: S \rightarrow S$, factors as $\pi \circ i$ where $i: S \rightarrow X$. If $f \in \Gamma\left(D_{+}\left(x_{j}\right), \pi_{*} O_{X}\right)$ is described as $f=\sum a_{l} Z_{j}^{l}$, $0 \leq l<p$, then $i^{*} f=\sum a_{l}^{p} i^{*}\left(Z_{j}\right)^{l}=\sum a_{l}^{p} g_{j}^{l}$ where $g_{j}=G / x_{j}^{p e} \in \Gamma\left(D_{+}\left(x_{j}\right), O_{S}\right)$. Thus in terms of homogeneous coordinates $i$ can be viewed on $O_{S}(-l e)$ as the composite

$$
O_{S}(-l e) \stackrel{F_{S}^{*}}{\rightarrow} O_{S}(-p l e) \stackrel{G^{l}}{\rightarrow} O_{S} .
$$

Received by the editors January 23, 1985 and, in revised form, July 8, 1985.

1980 Mathematics Subject Classification. Primary 14J05, 14F20.

${ }^{1}$ Research partially supported by an EPSCOR grant at IHES and University of Arkansas. 
Next we turn to the computation of $\pi_{*} \Omega_{X / S}^{1}$. On $D_{+}\left(x_{j}\right)$,

$$
\left.\pi_{*} \Omega_{X / S}^{1}\right|_{D_{+}\left(x_{j}\right)}=\bigoplus_{l=0}^{p-1} O_{S} \mid Z_{j}^{l} d Z_{j}
$$

and $d=d_{X / S}$ has $\bigoplus O_{S} \mid Z_{j}^{l} d Z_{j}, 0 \leq l<p-1$, as image. Since $Z_{j} x_{j}^{e}=Z_{k} x_{k}^{e}$, we conclude that

$$
\pi_{*} \Omega_{X / S}^{1}=\bigoplus_{l=1}^{p} O_{S}(-l e)
$$

and elements are locally given by $\sum a_{j} Z_{j}^{l} d Z_{j}$ where $d Z_{j}$ is a local representation of a section of $O_{S}(-e)$. Since $\Omega_{X / S}^{1}$ is an invertible $O_{X}$-module, all one forms are closed. [4].

We can now put all of this information together into the Cartier-Yuan sequence

$$
\begin{aligned}
& 0 \rightarrow G_{m, S} \rightarrow \pi_{*} G_{m, X} \stackrel{d l n}{\rightarrow} \pi_{*} \Omega_{X / S}^{1} \stackrel{C_{-} I}{\rightarrow} i^{*} \Omega_{X / S}^{1} \rightarrow 0, \\
& \pi_{*} \Omega_{X / S}^{1}=\bigoplus_{l=1}^{p} O_{S}(-l e), \quad i^{*} \Omega_{X / S}^{1}=O_{S}(-p e), \\
& C\left(\bigoplus_{l=1}^{p-1} O_{S}(-l e)\right)=0, \quad C: O_{S}(-p e) \rightarrow O_{S}(-p e) \text { is identity, } \\
& I \mid: O_{S}(-l e) \rightarrow O_{S}(-p e) \text { is the composite, } \\
& O_{S}(-l e) \stackrel{F_{S}^{*}}{\rightarrow} O_{S}(-p l e) \stackrel{G^{l-1}}{\rightarrow} O_{S}(-p e) .
\end{aligned}
$$

Note that $C$ is $O_{S}$-linear while $I$ is $p$-linear. This sequence is exact for the étale topology on $S$ and remains so when restricted to any subscheme of $S$.

We can now state our principal theorem.

THEOREM 1. Let $S$ be a regular, quasi-projective scheme in characteristic $p>$ 0 . Let $\pi: X \rightarrow S$ be the Zariski scheme associated to $(S, G)$ and assume $X$ is normal.

(1) Suppose $S$ is projective and $H^{m}\left(S, O_{S}(-n)\right)=0$ for $n>0$ and $m=0$ or 1. Then $\pi^{*}: \operatorname{Pic}(S) \rightarrow \operatorname{Pic}(X)$ is an isomorphism. If, moreover, $H^{2}\left(S, O_{S}(-n)\right)=$ 0 for $n>0$, then $\pi^{*}: \operatorname{Br}^{\prime}(S) \rightarrow \operatorname{Br}^{\prime}(X)$ is an isomorphism where $\operatorname{Br}^{\prime}(T)=$ $H^{2}\left(T_{e t}, G_{m}\right)$ is the cohomological Brauer group of $T$. Finally, if $S=\mathbf{P}_{k}^{2}, k a$ separably closed field, then $\operatorname{Pic}(X)=Z$ is generated by $O(1)$ and if $e=1$, then $\operatorname{Br}(X)=0$.

(2) Suppose $S=\operatorname{Spec} A$ is an affine, regular scheme. Then $\pi^{*}: \operatorname{Br}(S) \rightarrow \operatorname{Br}(X)$ is onto. If $S$ has no nontrivial Galois coverings with group $Z / p Z$ and either $G$ is $a$ unit in $A$ or $A$ is a factorial local ring with $G$ in the maximal ideal of $A$, then $\operatorname{Br}(S) \cong \operatorname{Br}(X)$ and $\operatorname{Pic}(S) \rightarrow \operatorname{Pic}(X)$ is onto. If $\operatorname{dim} A-\operatorname{dim} \operatorname{Sing}(X)>2$, then $X$ is locally geometrically factorial, i.e., $O_{X, p}^{h s}$ is factorial for all $p \in X$ where $O_{X, p}^{h s}$ is the strict henselization of the local ring $O_{X, p}$. In particular, $X$ is a locally factorial scheme.

Proof. Let $C=\operatorname{Cok}\left(G_{m, S} \rightarrow \pi_{*} G_{m, X}\right)$. Then we have two long exact sequences

$$
\cdots \rightarrow H^{r-1}(S, C) \rightarrow H^{r}\left(S, G_{m}\right) \rightarrow H^{r}\left(X, G_{m}\right) \rightarrow H^{r}(S, C) \rightarrow \cdots
$$


and

$$
\begin{aligned}
\cdots \rightarrow H^{r-1}(S, O(-p e)) & \rightarrow H^{r}(S, C) \\
& \rightarrow \bigoplus_{l=1}^{p} H^{r}(S, O(-l e)){\stackrel{C^{r}-I^{r}}{\rightarrow}}^{r} H^{r}(S, O(-p e)) \rightarrow \cdots
\end{aligned}
$$

where $H^{r}\left(S, \pi_{*} G_{m, X}\right)=H^{r}\left(X, G_{m}\right)$ since $\pi$ is finite and $C^{r}, I^{r}$ denote the actions of $C, I$ on the $r$ th étale cohomology groups $=r$ th Zariski cohomology of the coherent sheaves $O(-l e), 1 \leq l \leq p$.

(1) If $H^{0}(S, O(-n))=H^{1}(S, O(-n))=0$ for $n>0$, then $H^{0}(S, C)=H^{1}(S, C)$ $=0$ and so $\pi^{*}: \operatorname{Pic}(S) \rightarrow \operatorname{Pic}(X)$ is an isomorphism. If, in addition, $H^{2}(S, O(-n))=$ 0 for $n>0$, then $H^{2}(S, C)=0$, and so $\pi^{*}: \mathrm{Br}^{\prime}(S) \rightarrow \mathrm{Br}^{\prime}(X)$ is an isomorphism.

Finally, if $S=\mathbf{P}_{k}^{2}$, then $\operatorname{Pic}(S) \cong \operatorname{Pic}(X)$ since $H^{m}\left(\mathbf{P}^{2}, O(-n)\right)=0$ for $n>0$ if $m=0$ or 1 . Since $H^{2}\left(X, G_{m}\right)$ is torsion, $\operatorname{Br}(X)=H^{2}\left(X, G_{m}\right)[\mathbf{5}] . H^{2}\left(\mathbf{P}^{2}, G_{m}\right)=$ $H^{3}\left(\mathbf{P}^{2}, G_{m}\right)=0$ since we are over a separably closed field. Consequently, $\operatorname{Br}(X)=$ $H^{2}\left(\mathbf{P}^{2}, C\right)$ and if $e=1$, we arrive at the exact sequence

$$
0 \rightarrow \operatorname{Br}(X) \rightarrow \bigoplus_{l=1}^{p} H^{2}\left(\mathbf{P}^{2}, O(-l)\right) \stackrel{C^{2}-I^{2}}{\rightarrow} H^{2}\left(\mathbf{P}^{2}, O(-p)\right)
$$

There is a quite useful, concrete way of viewing $H^{2}\left(\mathbf{P}^{2}, O(-n)\right)$ (for example, see [3]). Consider the $Z$ graded ring $S_{X_{0} X_{1} X_{2}}=k\left[X_{0}, X_{1}, X_{2}\right]\left[\left(X_{0} X_{1} X_{2}\right)^{-1}\right]$. Then there is a graded isomorphism between $H^{2}\left(\mathbf{P}^{2}, \bigoplus O(n)\right)$ and the $k$ vector space in $S_{X_{0} X_{1} X_{2}}$ with basis $\left\{X_{0}^{l_{0}} X_{1}^{l_{1}} X_{2}^{l_{2}} / l_{i}<0\right.$ for $\left.i=0,1,2\right\}$. Thus a typical element $z \in \bigoplus_{l=1}^{p} H^{2}\left(\mathbf{P}^{2}, O(-l)\right)$ is

$$
z=\sum_{l=1}^{p} \sum_{\alpha} a_{\alpha}(l) M_{\alpha}(-l), \quad a_{\alpha}(l) \in k,
$$

where $M_{\alpha}(-l)=X_{0}^{l_{0}} X_{1}^{l_{1}} X_{2}^{l_{2}}$ with $l_{0}, l_{1}, l_{2}<0$ and $l_{0}+l_{1}+l_{2}=-l$. The description in (1.1) shows that

$$
\begin{aligned}
\left(C^{2}-I^{2}\right)(z)= & \sum_{\alpha} a_{\alpha}(p) M_{\alpha}(-p)-\sum_{\alpha} a_{\alpha}(p)^{p} G^{p-1} M_{\alpha}(-p)^{p} \\
& -\sum_{l=1}^{p-1} \sum_{\alpha} a_{\alpha}(l)^{p} G^{l-1} M_{\alpha}(-l)^{p} .
\end{aligned}
$$

Suppose $\left(C^{2}-I^{2}\right)(z)=0$. Multiply this equation by $\left(X_{0} X_{1} X_{2}\right)^{p(p-2)}$ to clear denominators. We then get the identity

$$
\begin{aligned}
& \left(X_{0} X_{1} X_{2}\right)^{p(p-2)}\left(\sum a_{\alpha}(p) M_{\alpha}(-p)\right) \\
& \quad=G^{2}\left(X_{0} X_{1} X_{2}\right)^{p(p-2)}\left[\sum_{l=3}^{p} G^{l-3} a_{\alpha}(l)^{p} M_{\alpha}(-l)^{p}\right] .
\end{aligned}
$$

In particular, $G^{2} \mid\left(X_{0} X_{1} X_{2}\right)^{p(p-2)}\left(\sum a_{\alpha}(p) M_{\alpha}(-p)\right)$, which is impossible unless $a_{\alpha}(p)=0$ for all $\alpha$ since $\operatorname{deg} G^{2}=p^{2}$ is too large. The remaining sum on the right must also be zero, and so we conclude that $\operatorname{Br}(X)=0$.

(2) Now suppose $S=\operatorname{Spec} A$ is an affine regular scheme. Let $X=\operatorname{Spec} B$ be the Zariski scheme associated to $(S, G)$ for a $G \in A$ and assume that $B$ is normal. 
Then $H^{r}\left(S, O_{(n)}\right)=0$ if $r>0$. This together with the sequence (1.3) allows us to conclude that

$$
0 \rightarrow H^{0}(S, C) \rightarrow \bigoplus_{l=0}^{p-1} A Z^{l} d Z \stackrel{C_{-} I}{\rightarrow} A i^{*} d Z \rightarrow H^{1}(S, C) \rightarrow 0
$$

is exact where $C$ is $A$-linear with $C\left(Z^{l} d Z\right)=0$ if $l<p-1$ and $i^{*} d Z$ if $l=p-1$ and $I$ is $p$-linear with $I\left(Z^{l} d Z\right)=G^{l} i^{*} d Z$. Consequently, $\operatorname{Br}(S) \rightarrow \operatorname{Br}(X)$ is onto.

Next we must analyze the cokernel of $C-I$. Suppose $b \in A$. Then $b i^{*} d Z=$ $(C-I)\left(\sum a_{l} Z^{l} d Z\right)$ iff we can solve the equation

$$
a_{p-1}^{p} G^{p-1}-a_{p-1}+\sum_{l=0}^{p-2} a_{l}^{p} G^{l}+b=0 .
$$

If we multiply this equation by $G$, set $a_{l}=0$ for $0 \leq l \leq p-2$ and $a_{p-1} G=T$, we arrive at the Artin-Schreier equation $T^{p}-T+b G=0$. Let $y \in A$ be a solution of this equation. If $G$ is a unit in $A$, then $a_{p-1}=y / G, a_{l}=0,0 \leq l \leq p-2$, is a solution of (1.4). Otherwise we factor $y^{p}-y=y(y-1) \cdots(y-(p-1))$ and observe that we may assume $y-1, \ldots, y-(p-1)$ are units. Since $A$ is factorial we conclude that $G \mid y$, and so $a_{p-1}=y / G, a_{l}=0,0 \leq l \leq p-2$, is again a solution of (1.4). In either case, $H^{1}(S, C)=0$, and so $\operatorname{Br}(S) \cong \operatorname{Br}(X)$ and $\operatorname{Pic}(S) \rightarrow \operatorname{Pic}(X)$ is onto.

Finally, let $W=\operatorname{Sing} X$ and suppose $\operatorname{dim} A-\operatorname{dim} W>2$. If $P \in X$ and $\pi(P)=Q$, then we can assume $A$ is strictly local since it is contained in the strict henselization at $Q$. Since there is only one point over $Q, B=A[Z] /\left(Z^{p}-G\right)$ is also strictly local. Then $\operatorname{Pic}(X-W)=\mathrm{Cl}(X)$, and we must show that this group vanishes. Let $U=S-\pi(W)$ and $V=\pi^{-1}(U)$. The sequence (1.1) for the mapping $\pi: V \rightarrow U$ remains exact. $S$ is regular and $\operatorname{depth}(\pi(W))=\operatorname{dim} A-\operatorname{dim} \pi(W)>2$. Hence $H_{\pi(W)}^{2}(S, A)=0=H^{1}\left(U, O_{S}\right)[\mathbf{2}]$. Moreover, the depth condition ensures that $H^{0}\left(U, O_{S}\right)=A$ and so just as above we get $H^{1}(U, C)$ via the exact sequence

$$
\bigoplus_{l=0}^{p-1} A Z^{i} d Z \stackrel{C_{-} I}{\rightarrow} A i^{*} d Z \rightarrow H^{1}(U, C) \rightarrow 0 .
$$

But we analyzed the image of $C-I$ above. Since $A$ is factorial and a separably closed local ring, the argument above applies and shows that $\operatorname{Pic}(U) \rightarrow \operatorname{Pic}(V)$ is onto. Since $A$ is factorial, $\mathrm{Cl}(A)=\operatorname{Pic}(U)=0$ and so $\mathrm{Cl}(B)=0$.

Let $k$ be separably closed and let $X$ be a normal Zariski surface associated to $\left(\mathbf{P}_{k}^{2}, G\right)$ where $G \in \Gamma\left(\mathbf{P}^{2}, O(p e)\right)$. Let $p: \tilde{X} \rightarrow X$ be the minimal desingularization of $X$. The last calculation we want to make concerns the Picard and Brauer group of the desingularized Zariski surface $\tilde{X}$. It can be shown that $X$ has only rational double point singularities with resolution graph $A_{p-1}$ [1]. If $T \subseteq X$ is the discrete singular set, let $W=p^{-1}(T)$.

THEOREM 2. If $\tilde{X}$ is a desingularized Zariski surface as above, then $\operatorname{Pic}(\tilde{X})$ is a free abelian group of rank $=(p-1) \#(T)+1$ and $\operatorname{Br}(X) \rightarrow \operatorname{Br}(\tilde{X})$ is onto. If $e=1$, then $\operatorname{Br}(\tilde{X})=0$.

PROOF. Since $p_{*} G_{m, \tilde{X}}=G_{m, X}$ and $\tilde{X}, X$ are proper, we have an exact sequence [6, Exercise 3.4, p. 229].

$$
\stackrel{\cdots}{\quad} \operatorname{Pic}(W) \rightarrow H^{2}\left(X, G_{m}\right) \rightarrow H^{2}\left(\tilde{X}, G_{m}\right) \rightarrow H^{2}\left(W, G_{m}\right) \rightarrow H^{3}\left(X, G_{m}\right) \rightarrow \cdots
$$


Now since $X$ has rational singularities, $\operatorname{Pic}(W)$ is a free abelian group whose cardinality is the number of irreducible components of $W$; that is, $(p-1) \#(T)$. By Theorem $1, \operatorname{Pic}(X)=Z$ and so $\operatorname{Pic}(\tilde{X})$ is free abelian of $\operatorname{rank} 1+(p-1) \#(T)$.

In order to finish the proof we need only show that $H^{2}\left(W, G_{m}\right)=0$. For this we use induction on the number of $\mathbf{P}^{1}$ 's appearing in $W$. If there is only one, then by Tsen's Theorem $H^{2}\left(\mathbf{P}^{1}, G_{m}\right)=0$. For the induction step we use the exact sequence (1.5) where we let $X$ be our $W$, and $\tilde{X}$ be obtained by blowing up a nonsingular point on $W$. Then $W$ becomes $\mathbf{P}^{1}$ and the exact sequence shows that $H^{2}\left(X, G_{m}\right)=H^{2}\left(\tilde{X}, G_{m}\right)$, as desired.

ACKNOWLEDGMENTS. The authors would like to thank O. Gabber and T. Ekedahl for several useful conversations and notes concerning this paper.

\section{BIBLIOGRAPHY}

1. P. Blass, Some geometric applications of a differential equation in characteristics $p>$ 0 to the theory of algebraic surfaces, Contemporary Math., Vol. 13, Amer. Math. Soc., Providence, R.I., 1982.

2. R. Hartshorne, Local cohomology, Lecture Notes in Math., vol. 41, Springer-Verlag, Heidelberg, 1967.

3.

4. R. Hoobler, Cohomology of purely inseparable Galois coverings, J. Reine Angew. Math. 66 (1974), 183-199.

5. _ When is $\operatorname{Br}(X)=\mathrm{Br}^{\prime}(X)$ ?, Brauer Groups in Ring Theory and Algebraic Geometry, Lecture Notes in Math., vol. 917, Springer-Verlag, New York, 1982, pp. 231-245.

6. Milne, Étale cohomology, Princeton Math. Series, no. 33, Princeton Univ. Press, Princeton, NJ., 1980.

Department of Mathematical Sciences, University of ARKansas, FayetteVILLE, ARKANSAS 72701

Department of Mathematics, City College of New York, NeW York, NeW YORK 10031 